\title{
Feasibility of vaccination against Macrobrachium rosenbergii nodavirus infection in giant freshwater prawn
}

\begin{abstract}
The giant freshwater prawn/giant river prawn, Macrobrachium rosenbergii is one of the high market value crustaceans cultured worldwide. The intensified aquaculture of the species has led to the outbreak of infectious diseases, prominently, the white tail disease (WTD). It is caused by the infection of Macrobrachium rosenbergii nodavirus (MrNV), which was classified in the family of Nodaviridae. To-date, there are no effective prophylactic and therapeutic agents available against MrNV infection. Vaccination is known to be the most effective prophylactic agent in disease prevention. However, vaccine development against virus infection in crustaceans is equivocal. The feasibility of vaccination in conferring immune protection in crustaceans against infectious diseases is disputable. The argument lies in the fact that crustaceans do not possess adaptive immunity, which is the main immune component that functions to establish immunological memory upon vaccination. Nevertheless, an increasing number of literatures has been documented, which concerns the development of vaccines against infectious diseases in crustaceans. The current review deliberates different approaches in vaccine development against MrNV, which were documented in the past years. It is noteworthy that the live-attenuated MrNV vaccine has not been experimented by far. Thus, the potential of live-attenuated MrNV vaccine in conferring long-term immune protection through the establishment of innate immune memory is currently being discussed.
\end{abstract}

Keyword: Macrobrachium rosenbergii; MrNV; Nodavirus; Vaccine; White tail disease 